\title{
Static Background Extraction and Updating Algorithm based on Adaptive Weights
}

\author{
Sang Haifeng*, Chen Yu, Chen Zhong \\ School of Information Science and Engineering, Shenyang University of Technology, \\ Shenyang 110870, China \\ * Corresponding author's Email: sanghaif@163.com
}

\begin{abstract}
Compared with several methods of extraction from static background of video, and for the limitations of these methods, a new type of method is put forward for updating background extraction. In this method background weight is thought as a standard of reliability to evaluation background by introducing a new parameter value and its variation, and is anticipated to maintain or upgrade the numerical size control background. For each frame image of two judges, the image elements will be classified, and the variation of background weights will be produced separately, control weight by weight change background. The experimental results show that this method has the advantages of simple principle, fast operation, updates faster and anti-interference ability of the foreground, and can be adapted to different circumstances in background extraction through adjusting the setting parameter.
\end{abstract}

Keywords: static background extraction; background weights; variation of background weights; background update.

\section{Introduction}

Computer vision monitoring is currently a hot research field in computer vision, and it gets more attention in recent years. In the system to computer vision, it is an important basic technology to pick moving-object image from the video sequence in real-time. Visual surveillance system, a frontier research direction in the field of computer vision in recent years, has broad application prospects of the movement in the traffic monitoring, dynamic environment in real time observation and automatic navigation system ${ }^{[1]}$. The static background target detection means that the camera has the static background as a reference, and it's usually used for monitoring fixed scene like road and so on ${ }^{[2]}$.

At present, there are three methods of moving object detection based on video: optical flow ${ }^{[3]}$, frame difference ${ }^{[4]}$ and background difference method ${ }^{[5]}$. Optical flow method can be used for camera motion, and it has complete information extraction of moving objects (including motion information), high computational complexity, poor anti-noise per- formance, and the powerful hardware is needed to support ${ }^{[6]}$. The inter frame difference method extracts moving objects using the difference between adjacent frames in a video sequence. The inter frame difference method has the advantage of less computation, strong robustness, and strong adaptability. The disadvantage is that it is difficult to extract the pixel point from the moving target, which is easier to produce void pixel ${ }^{[7]}$.

The background difference method, as a method widely used in motion detection, is a key part of computer vision, effective segmentation of background and prospects for the follow-up tracking, object recognition, and behavior classification. It plays a decisive impact in high-level processing ${ }^{[8]}$. It has the advantages of simple principle, faster algorithm, and extraction of moving objects completely and so on. There are two key problem of background difference method: how to extract a pure background image and how to update the background image.

In the video as an example, compared with the traditional method of extracting the background, a static background model extraction and update al- 
gorithm is proposed. With the introduction of new parameters, to avoid the interference between the prospects from the background, it has strong adaptability to the changes in the background. The result of experiment shows that this method is simple and effective.

\section{Basic method of background extraction}

According to the different focus, background extraction methods can be divided into two categories: the method based on model and the method based on the updating strategy. The former is focused on the establishment of a mathematical model to describe the difference between background and foreground information, and the background image can be extracted by means of it. The latter is focused on establishment method suitable for the scene update. The background image can be constantly updated to approach true background ${ }^{[9]}$. Traditional methods of background proceed around the two focuses of various methods, but due to various reasons, each one of them has no prominent advantage.

\subsection{The weighted average method}

The principle of weighted average method is to update the background image constantly by using the current frame image, and it is a cumulative process. This method is not required to accumulate a certain number of frames of the video image as the initial background, more time saved and faster speed of operation improved. Therefore it has more practicability based on simple and easier implementation. The weighted average method model is simple, easy to calculate, but are vulnerable to foreground background interference. When the moving targets become fewer or the movement speed is fast, you can get a better framing effect, but when the moving targets are more or moving at a slower speed, the background is shuttered by moving target in a long time. Foreground image will affect the background extraction. We call it "ghost".

\subsection{Surendra algorithm}

Surendra algorithm has the main idea of getting the two value images by inter frame difference, and selecting the pixels of the current frame for update and then update them selectively. The Surendra algorithm is greatly affected by the initial frame. If a moving target appears in the starting frame, the following background update will appear in the "ghost" and cause the deviation of the movement target detection afterwards. As a result it will lead to unsatisfactory or even erroneous results.

\subsection{Gauss mixture model method}

Gauss mixture model is modified on the Gauss model, whose main idea is to divide each pixel gray value sequence in time as a series of random variables. Multiple single Gauss (normal) distribution function is used to describe the multimodal background. This method of background extraction is more accurate, suitable for small change of background and fast moving target. Gauss mixture model method also has some shortcomings: sensitive to global changes in brightness and unsuitable for the moving target which is large, slow-moved, computationally complex and of poor real-time performance.

\section{Algorithm description}

In several common methods, although their update of the different methods is different from each other, but there are two main problems:

Firstly, the image read in will directly impact the background image extracted, the current frame image will more or less effect the background extraction, resulting in "ghost"; Secondly, if the current frame goes through a certain algorithm rules and not directly act on the background image extracted, you need to save some images for reservation, and it will hog up the most memory and lead to slowdowns.

This paper introduced a new algorithm in parameters, changes in the parameters are thought as the "buffer" between the current frame image and the background image, so as to control the output of the background with numerical parameters, non-ghost, no more image frame for reservation.

\subsection{Parameter definition}

The new parameters are introduced in the algorithm: background weight $w$ and background weight variation $p$. Background value of $w(\mathrm{x}, \mathrm{y})$ represents the degree of reliability that we consider the pixel position at $(\mathrm{x}, \mathrm{y})$ as the output background, the greater $w$ value is, the more reliability that the current pixel is considered as the output background. When the value of $w(\mathrm{x}, \mathrm{y})$ is positive, it indicates more reliable that current pixel is looked as the background output; if $w(\mathrm{x}, \mathrm{y})$ is 0 or negative, it indicates that the current pixel as output background is not reliable, it needs to update. Background weight corresponding to each pixel is independent, not affected by various parameters of the other pixels. As the image is composed of a matrix arranged 
by pixel in sequence, background value of each pixel consists of a matrix as the same. The initial value of $w(\mathrm{x}, \mathrm{y})$ is set to 0 .

Weight change $p_{k}(\mathrm{x}, \mathrm{y})$ represents the variation of weight $w(\mathrm{x}, \mathrm{y})$ of the pixel at point $(\mathrm{x}, \mathrm{y})$ position on the $\mathrm{k}$ frame image. Defined as the background value, background weight variation is also associated with each pixel. At each frame, the background weight variation corresponding to each pixel is independent. $p_{k}(\mathrm{x}, \mathrm{y})$ initial value is set to 1 . (All parametric variables below indicate it is corresponded to the pixel (x,y), ellipsis $(\mathrm{x}, \mathrm{y})$ )

\subsection{The basic idea of the algorithm}

We describe the reliable degree of the background pixel values correspondingly by introducing background weight, no more previous figures that have ever been read are needed to be stored, and the memory is released. To change the background weight as strain on the read image, avoid reading the image directly impact on the background image and put an end to the "ghost" problem. In addition, among the images that have been read, the image elements which have different effects on background extraction should be classified. We should classify the image for the second time. To be specific we should classify the current frame image elements, for the image elements of different classes and different control strategies based on different weights will be used with the image elements in different categories. Finally, we can make a decision if the background is more reliable and then make a final output of the background.

\subsection{The algorithm flow}

The first step: Read in video, if it is the first frame, the first frame image will be treated as the background.

The second step: Make a judgment that if the current frame $I_{k}$ is equal to the background $B_{k-1}$ in the last frame output, when they are the same, then the initial current frame $I_{k}$ will be considered as background elements.

The third step: Make a judgment that if the current frame $I_{k}$ is equal to $I_{k-1}$ in the last frame, when they are the same, it indicates that the current frame and the previous frame is coherent.

The fourth step: Through judgment of two or three steps above, the state of current frame is divided into four types. According to four different circumstances to determine the values of the background weight variation $p_{\mathrm{k}}$, the formula is as follows:

$$
p_{k}=\left\{\begin{array}{l}
\alpha p_{k-1}, I_{k}=I_{k-1}, \text { and }, I_{k}=B_{k-1} \\
\beta p_{k-1}, I_{k}=I_{k-1}, \text { and }, I_{k} \neq B_{k-1} \\
\delta, I_{k} \neq I_{k-1}, \text { and }, I_{k}=B_{k-1} \\
\varepsilon, I_{k} \neq I_{k-1}, \text { and }, I_{k} \neq B_{k-1}
\end{array}\right.
$$

Where $\alpha, \beta, \delta, \varepsilon$ is setting parameters according to the actual situation of choice, the selection strategy will be introduced in detail late on.

The fifth step: the previous background of weight $w_{k-1}$ will be adjusted by value of the background weights variation $p_{k}$, so we get the background weight $w_{k}$, the formula is as follows:

$$
W_{k}=w_{k-1}+p_{k}
$$

The sixth step: the background of $B_{k}$ will be determined by the value of the background weight $w_{k}$, the formula is as follows:

$$
B_{k}=\left\{\begin{array}{l}
B_{k-1}, w_{k}>0 \\
I_{k}, w_{k} \leq 0
\end{array}\right.
$$

The algorithm flow chart is shown in Figure 1.

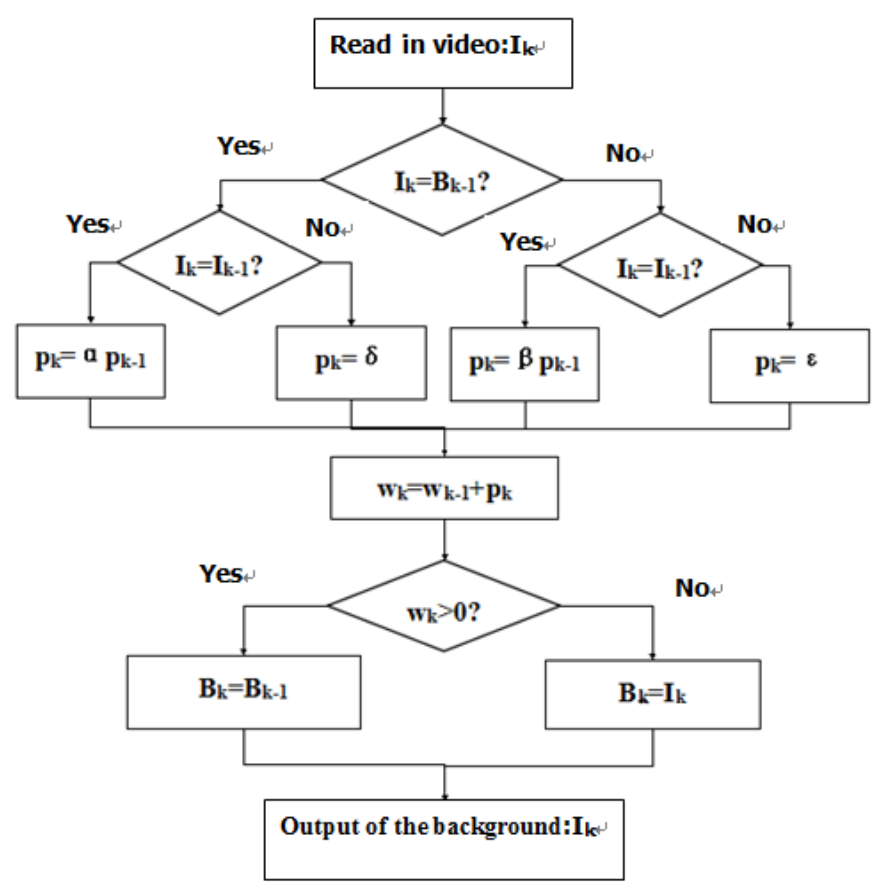

Figure 1 Flow chart of the algorithm

Updating strategy of the scheme is to classify the input image elements. Make a judgment whether the current frame image is equal to previous frame image and the previous frame background image. When it is equal to the output background of the previous frame, it will be determined as the back- 
ground elements. On the contrary, it will be thought as the target element or noise. If it is the same as the previous frame image, it represents "continuous image", otherwise it represents "discontinuity". By two decisions, the image information read in will be classified. The image information will be classified into different properties. According to the properties of different image elements, different changes were made to the background. Making two judgments, it can be divided into 4 cases in analysis:

(1) When the current frame, previous frame and the background of previous frame are equal ( $\alpha$ state), at this time because the background and previous frame are equal to the current frame, it can be identified as the background elements appeared continuously and the reliability of the background increased, therefore leads to the accumulation of $w . \alpha$ state is a continuous state, which is continuous process of accumulation in the background.

(2) The current frame and previous frame are equal, but is not equal to the background of previous frame ( $\beta$ state). If there is a short time, it can be identified as the moving target and the reliability of background slightly lowered. Then we can decrease the value $w$ slowly. If this state is keeping for a long time, it suggests that the background has already changed to a much lower reliability, and then we can decrease the value $\mathrm{w}$ rapidly. $\beta$ status is a continuous state. $\beta$ state upfront is a background with a moving target shuttered, and late $\beta$ state is the process of updating background.

(3) The current frame is not equal to previous frame, but when it's equal to the background of the previous frame ( $\delta$ state), we can consider that the moving target has left. The background appeared in the image again, because only when the static background elements are repeated many times in the video will it be very difficult for moving targets to appear for so many times and will it accumulate as a background. Because only the background elements have the characteristics of appearing repeatedly, so reliability of the background will be promoted sharply, to boost $w$. If the gray value appears continuously, it will change to $\alpha$ state. When $\delta$ state is an instantaneous state and obscure in the background again, it provides the initial value of the background weight variation for the $\alpha$ state.

(4) The current frame is not equal to previous frame, and it is not equal to the background of the previous frame ( $\varepsilon$ state). At this moment the gray value and the gray value of the previous frame is not a continuous state, and it is different from backgrounds. So at this time it will be looked as a noise. In addition, when noise target is moving, this kind of situation can appear as much. If the gray value pre- sents continuously after that and goes to the $\beta$ state, $\varepsilon$ state is a transient state.

The adaptive background weights is mainly manifested that after the initial set up of background values and its variation, subsequent background weight and its changes will alternate by itself as images read in video frame by frame. There is no need to control background weights and its variation.

This paper proposed a new algorithm. Compared with the traditional algorithm, the biggest difference is that it uses movement type update when updating background. Traditional algorithm uses gradient type update, in which foreground element will always more or less influence the extraction of the background, resulting in a virtual shadow. When the background needs to be updated and namely background weights $w$ is zero or negative, the new method directly lets the background update for the current state, namely dances background update, and omits the gradient update process. Within a short time after changing the background, it achieves the updating background in one step.

\section{$3.4 \alpha, \beta, \delta, \varepsilon$ epsilon value strategy}

The four custom parameters $\alpha, \beta, \delta, \varepsilon$ are constant value of parameters set for the characteristics of specific environment and the moving object, which can be adjusted according to the specific requirements. The four parameters have a certain value strategy respectively.

\subsubsection{Parameter $\alpha$}

Parameter $\alpha$ appears in the circumstance that the current frame is equal to previous frame and the output background in previous frame ( $\alpha$ state). Because the current frame and its background have no change to the previous frame, it can be regarded as the "continuous" elements of background, weight $w$ of background accumulates. As many frames read in, $w$ value gets accumulation and the $w$ value increases, which indicates the high reliability of the output background.

Continuously reading in the same gray value can make the $\mathrm{w}$ value continuous increase. If $w$ value increases rapidly, it will make the w value too high to eliminate. The beginning of the video, for example, a moving target image will come out just for a temporary rest. With the time passing the moving targets start to move. In its original area gray value of moving target accumulates to high $w$ value and frames are required to eliminate the increase of $w$ value, resulting in a little longer for updating the regional background. Furthermore, when the back- 
ground changed, high $w$ value will also lead to the slow background update. Thus, the cumulative rate of $w$ value must be properly controlled, so the $p$ value should continue to decline, and the cumulative rate of the $w$ value tends to slow down with time going on, as shown in Figure 2.

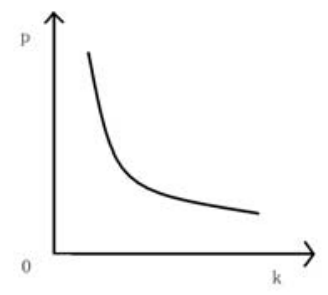

Figure $2 p$ value changing trend under $\alpha$ state

With the time going on, $p$ value decreases, decreases slowly. So $\alpha$ value should be less than 1 . According to the different environment, if the number of moving target is large or its speed is slow, the time of the emergence of the background is short, and then $\alpha$ value should increase. Otherwise $\alpha$ value decreases. According to the experience, $\alpha$ value usually takes $0.96 \sim 0.99$.

\subsubsection{Parameter $\beta$}

The situation with $\beta$ parameters emerged is that the image of the current frame and the image of the previous frame is the same, but different with the background output of the previous frame ( $\beta$ state). $\beta$ state is divided into two stages. The initial tentative in this area is solid color moving target elements, which make the subtraction of background weight $w$. In a short period of time, the deduction of $w$ value does not cause the $w$ value to be negative. The late frames for a large number repeat the same gray value, suggesting that this region may need to update the background. The gray value still appears continuously and the value of $w$ decreases continuously. Finally when $w \leq 0$, the current frame becomes the background, comes back to the case in $\alpha$ state, and starts background accumulation. Thus, $\beta$ parameter will not only ensure the initial $w$ value of $\beta$ state will decrease slowly, but also ensure the $w$ value during the late $\beta$ state can be quickly reduced, as shown in Figure 3 .

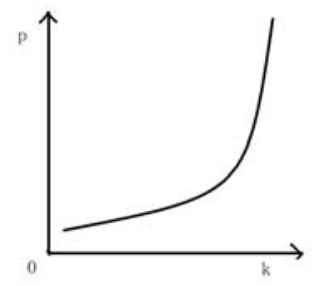

Figure $3 p$ value changing trend under $\beta$ state
As the time goes by, the $p$ value increases, and the growing rate is faster. In a short time the reduction of $w$ value will not have a great influence on the $w$ value, so it will maintain the original background output as usual. But with time $p$ increasing, it leads to $w$ value decrease rapidly, and ultimately achieves the purpose of background updating. $\beta$ value should be greater than 1 . When the target number or movement speed gets slow, $\beta$ value can be properly reduced. Otherwise the $\beta$ value increases. According to the experience, $\beta$ value usually takes $1.02 \sim 1.05$.

\subsubsection{Parameter $\delta$}

$\delta$ parameter appears in the case that the current frame is different from the previous frame but the same as the output background in previous frame. At this time it can be considered that the background elements appear again. Value $\delta$ will take a large fixed value. If the gray value remains the same as the value of the output in previous frame and repeated, it returns to the astate. Background elements have the only features with multiple occurrences. With these characteristics the background gets high degree of confidence. Background value $w$ embodied in the "reliability" acts as the background parameters, so background value $w$ needs to be increased sharply. $\delta$ is set to a larger constant value, which can provide a larger $p$ value for $\alpha$ status, thus the speed accumulated in background weight $w$ will be greatly enhanced. Once $\delta$ state appears, it will improve the overall efficiency of an accumulation of $w$ value in $\alpha$ state. Compared to $\alpha, \delta$ value is also more in moving target number or moving slowly. In that case $\delta$ value increases. Conversely, $\delta$ value decreases. Empirical values of $\delta$ is $1.5 \sim 2$.

\subsubsection{Parameter $\varepsilon$}

$\varepsilon$ parameter appears in case that the current frame and the previous frame image is unequal, but unequal to the output background of the previous frame, then it will be regarded as noise or noise of moving targets. Whether it is noise or noise targets, it should not be interfered to the background, so the background weights are slightly reduced. When the weight variation of $p$ is close to the smaller numerical 0 , the original background weight does not change and the value of $w$ remains basically unchanged. If the number of subsequent frames and the current frames are the same, it will go into $\beta$ state. $\varepsilon$ value is thought as the initial value of the background variation of $p$ and it continues to reduce the background weight $w$ until the background is re- 
placed. If there is noise at this time, it will immediately turn into $\delta$ State, and then to $\alpha$ state, which promotes the accumulation of background weight, makes the background output becomes more stable and indicates that the method has strong anti-noise ability. Empirical value $\varepsilon$ is $-0.02 \sim-0.01$.

\subsection{Insensitive weight variation processing}

The judgment method applies for the condition when several image gray values are equal, for small fluctuations in brightness in static background lead to subtle changes the gray value and then result in accumulation of chaos scheme background weight $w$. In the actual environment it may not appear very stable phenomenon of gray value, thus the weight variation $p$ will be the following insensitive processing:

$$
p_{k}=\left\{\begin{array}{l}
\alpha p_{k-1},\left|I_{k}-I_{k-1}\right| \leq M, \text { and },\left|I_{k}-B_{k-1}\right| \leq M \\
\beta p_{k-1},\left|I_{k}-I_{k-1}\right| \leq M, \text { and },\left|I_{k}-B_{k-1}\right|>M \\
\delta,\left|I_{k}-I_{k-1}\right|>M, \text { and },\left|I_{k}-B_{k-1}\right| \leq M \\
\varepsilon,\left|I_{k}-I_{k-1}\right|>M, \text { and },\left|I_{k}-B_{k-1}\right|>M
\end{array}\right.
$$

$M$ is set to the fluctuation allowed to change, which can be adjusted according to actual situation. $M$ usually takes $2 \sim 5$. The operator "I |" means the absolute value.

After insensitive processing, subtle changes of grayscale image will not cause weight accumulating chaotic to the background.

\section{Experimental verification}

We take something indoor as shooting situations, and select a few representative movements as sample. System program is prepared using $\mathrm{VC}++6.0$ and OPENCV1.0.

\subsection{The work flow}

Firstly, the workflow of the proposed algorithm will be showed in this paper through an example. Video content: testing personnel get into the picture on the right, step forward for a few paces then make a short stay (about 60 frames), continue to move forward, after a few steps forward again make a long time stay (about 400 frames), then go back along the original road out of the picture(About at 80th frames get into the picture, about at 140th frames make a short stay for the first time, about at 210th frames continue to move forward, about at 300th frames create a second residence for a long time, about at 720th frames along the original road return, at 780th frames completely get off the screen).

\subsubsection{Initialization}

First to read in the video, we just take the first image as the initial background image. The initial background weight $w$ to each pixel value is 0 . The initial background weight variation $p$ of each pixel is 1. At this point the first frame of the video will directly output as the background, as in Figure 4.

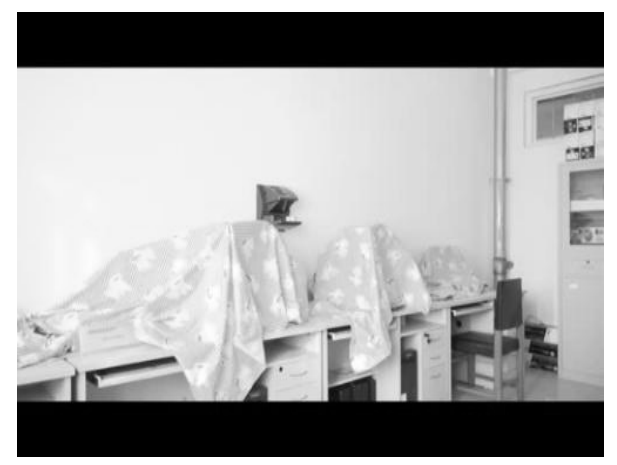

Figure 4 Initial background

\subsubsection{Moving target}

About at 80th frames, testing personnel get into the picture. Before 80th frames, we take the first frame as the background, and then continue to read in the video. Images after it are almost the same with the image of the first frame, therefore it enters the $\alpha$ state and background value $w$ of each pixel keep accumulating. Due to the control of $\alpha$ coefficient, the speed of accumulation of $w$ background weight gets slowly. But $w$ keeps increasing, the $w$ value is positive and it is still the original background. Because the image has not changed, it always outputs a first frame image as the background, as shown in Figure 5.

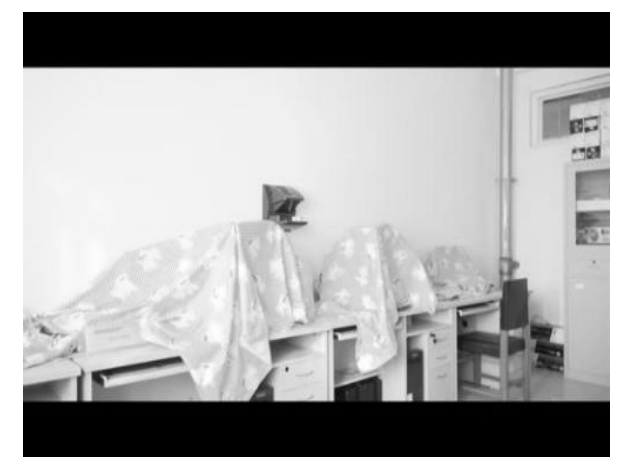

Figure 5 Image of background from 80th frames

After 80th frame, testing personnel get into the 
picture as the moving target, and then make a short stay. Moving object first enters the edge of the region. Due to the entering of the moving target, this $r-$ egion goes into instantaneous $\varepsilon$ state, the moving target is considered as noise, background value $w$ corresponding to pixel in the area gets small deduction, and $w$ does hardly change. After the entering of moving target, regardless of the motion target motion and short stay, moving target continues to exist in the region and pixel in the region goes into the early $\beta$ state. The $\beta$ state is given a smaller initial value by state $\varepsilon$ state, so the background weight $w$ reduces slowly. Although the $w$ decreases, it remains positive, so the background is not updated and still maintains the original background output. While the area passes through by moving target, in the moment of the moving target leaving from the region, the pixel of the area goes into instantaneous $\delta$ state. Since the background repeatedly appears, a substantial accumulation is made with the background value $w$. Due to the continuous emergence of the background, this area pixels goes into the $\alpha$ state. Because the $\delta$ state before has given greater initial value to the $\alpha$ state, the accumulation speed of background weight $w$ in $\alpha$ state increases, $w$ continues to accumulate, background continues to output the original background, as shown in Figure 6.

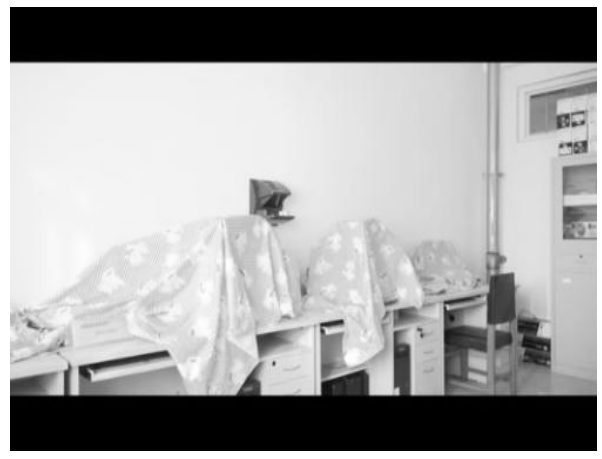

Figure 6 Background image in 180th frame

\subsubsection{Background update}

Moving target in a region remains static for a long time. Moving object is persistent in static region, by instantaneous $\varepsilon$ state to state first during the early $\beta$ state. Background weight $w$ slightly reduces, $w$ remains positive, so the background to the original background continuously outputs; Moving target is stationary for a long time, and this area accesses $\beta$ state later. At this time the background weighted $w$ substantially reduces. Until a certain time the background value $w$ is close to 0 or negative, then the current frame image as the background is to lose.
The testers instantly appear in the background image as moving target, as shown in Figure 7.

After the moving target has a rest for a period of time, the moving target of the background in static background motion background image target has been updated. Leave the video screen immediately, the original target is in static region for a long period. It begins to enter the instantaneous $\varepsilon$ state, changes to early $\beta$ state first, and then goes into $\beta$ state later. Background weight $w$ of pixels corresponding to the moving target is reduced to 0 or negative. Moving target disappears from the background image and background updates again. When the target appears in the background image the accumulation background weights of $w$ become smaller. After a small number of frames, it is updated again, as shown in Figure 8 .

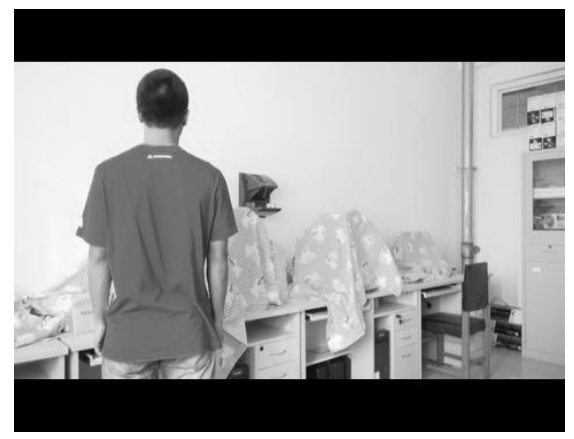

Figure 7 Background image in 720th frame

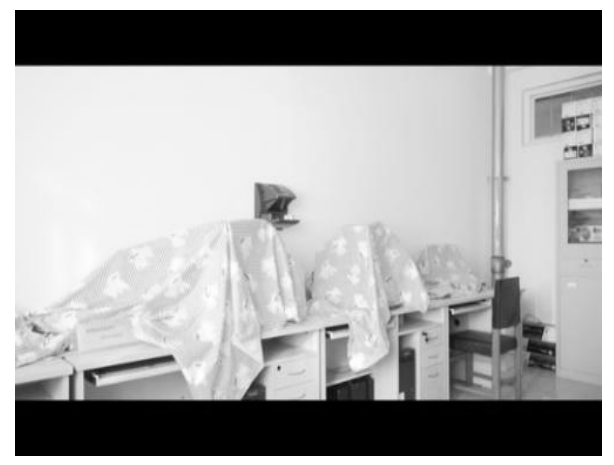

Figure 8 background image in 800th frame

\subsection{Ghost experiment}

Testers enter the picture from the right, go back and leave after a brief stay (about at 550th frames go into the picture, about 700th frame stop place to stay. To leave the picture along the way, about 980th frames of about 1050th frames leave picture).

Through the experiment, we can see, multi frame average method and the Surendra algorithm will be interfere in the view. Especially when speed gets 
slow or stops for a while the ghost will gradually become real. Anti-interference for the foreground elements is insufficient. Gauss model accumulates for a period of time, forms a relatively stable Gauss's model. Anti-interference ability for the foreground elements is very strong. As long as it is accumulated over a period of time in advance, the Gauss model is an excellent framing method with the strong antiinterference.

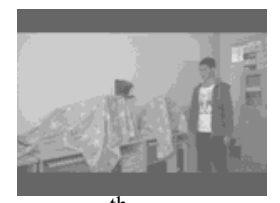

a) $600^{\text {th }}$ frame b) $720^{\text {th }}$ frame

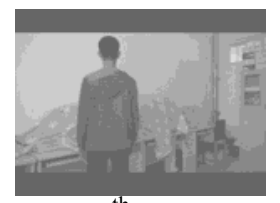

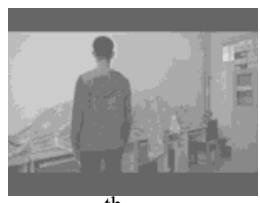

c) $840^{\text {th }}$ frame

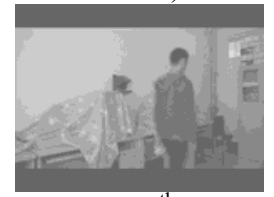

d) $1000^{\text {th }}$ frame

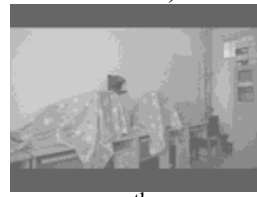

e) $1200^{\text {th }}$ frame
Figure 9 The $600^{\text {th }}, 720^{\text {th }}, 840^{\text {th }}, 1000^{\text {th }}, 1200^{\text {th }}$ frame image in original video

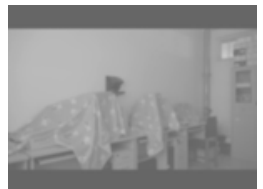

a) $600^{\text {th }}$ frame

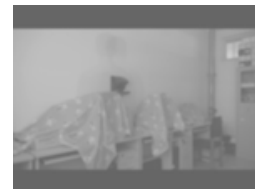

b) $720^{\text {th }}$ frame

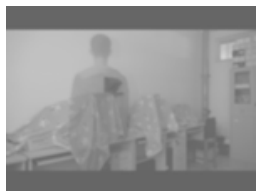

c) $840^{\text {th }}$ frame

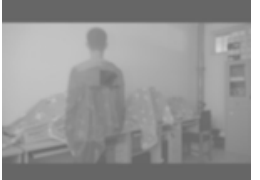

d) $1000^{\text {th }}$ frame

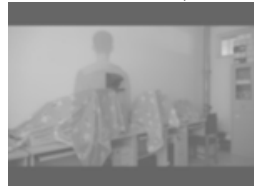

e) $1200^{\text {th }}$ frame
Figure 10 The 600th, 720th, 840 th, 1000 th, 1200 th frame image in multi frame average method

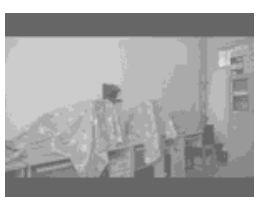

a) $600^{\text {th }}$ frame

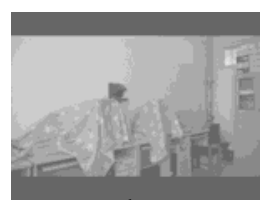

b) $720^{\text {th }}$ frame

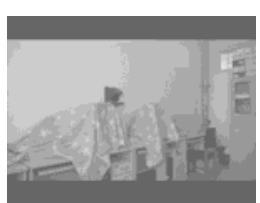

c) $840^{\text {th }}$ frame

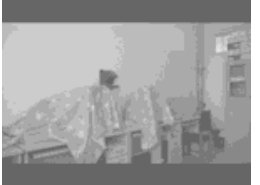

d) $1000^{\text {th }}$ frame

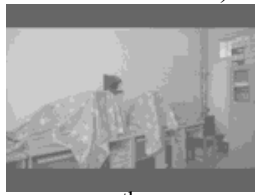

e) $1200^{\text {th }}$ frame
Figure 11 The $600^{\text {th }}, 720^{\text {th }}, 840^{\text {th }}, 1000^{\text {th }}, 1200^{\text {th }}$ frame image in Gauss model

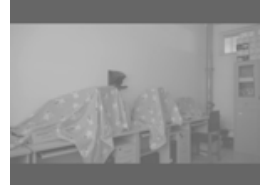

a) $600^{\text {th }}$ frame

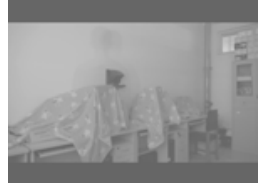

b) $720^{\text {th }}$ frame

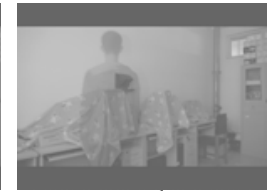

c) $840^{\text {th }}$ frme

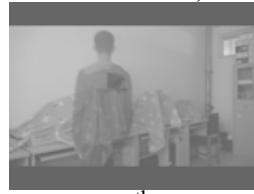

d) $1000^{\text {th }}$ frame

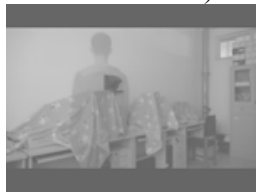

e) $1200^{\text {th }}$ frme

Figure 12 The $600^{\text {th }}, 720^{\text {th }}, 840^{\text {th }}, 1000^{\text {th }}, 1200^{\text {th }}$ frame image in Surendra algorithm

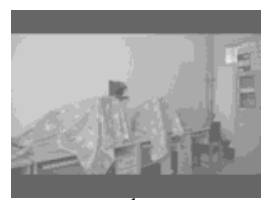

a) $600^{\text {th }}$ frame

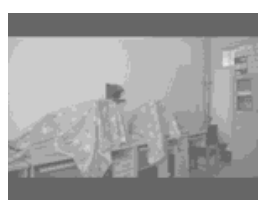

b) $720^{\text {th }}$ frame

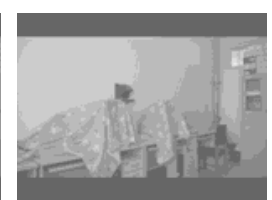

c) $840^{\text {th }}$ frame

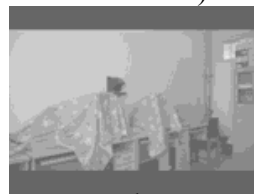

d) $1000^{\text {th }}$ frame

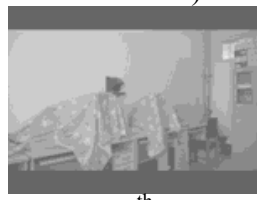

e) $1200^{\text {th }}$ frame
Figure 13 The $600^{\text {th }}, 720^{\text {th }}, 840^{\text {th }}, 1000^{\text {th }}, 1200^{\text {th }}$ frame image in our methods

The method proposed in this paper, firstly is to cumulate on the background, then go into the $\alpha$ state, cumulate on background and make an accumulation on weight $w$ of the background. $w$ value is increasing. After moving target enters, $w$ value to the passed location decreases. As a short time passes by a stop will go into the $\beta$ state prophase, which will not cause the $w$ value reduce below 0 . The background will not update, which shows the strong resistance against the foreground interference, and it is in no way inferior to Gauss model.

\subsection{Update speed experiment}

Testers start standing in the picture, after a period of time, leave quickly off the screen (start to stand for about 750th frame, for 820th frame completely off the screen, 1653 frames in total).

In the experiments from the above, the moving targets still appear on the start screen, and then stay for a long time. All methods will make accumulation with the background which considers the moving object remaining in the picture from the initial.

After moving target left, the multi frame average method and Surendra algorithm method of initial moving target begins to become empty, fade, which indicates the Surendra algorithm, is affected by the 
initial impact. Gauss model method has accumulated a relatively stable Gauss model due to the target initially residence. After the target moves away the image of motion object extracted in the background of the Gauss model still maintains a long period of time. As time passes it disappears slowly. Gauss model has poor update ability especially for moving targets which remain on the screen for a long time just from the start.

The method in this paper is to make accumulation to the background as the traditional method, and $w$ value increases. After moving target leaves from the picture, $w$ value of the initial position of moving object decreases. Through transient $\varepsilon$ phase into the $\beta$ stage, the $w$ value decreases slowly. Background maintains the original state. With the time passing it begins to enter the $\beta$ later stage and $w \mathrm{v}-$ alue decreases rapidly. In about 1150 frames, the moving object in the background disappears and realizes the leap type update background.

The update speed may accelerate by adjusting $\beta$ parameter and $\varepsilon$ parameters.

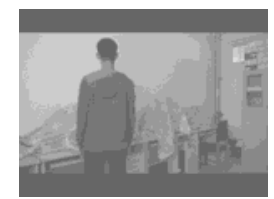

a) 500th frame

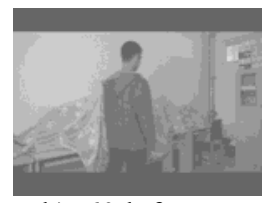

b)760th frame

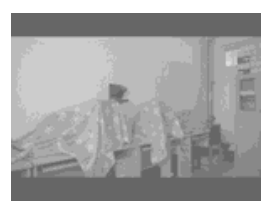

c)1000th frame

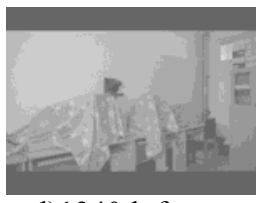

d)1240th frame

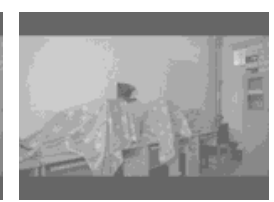

e)1500th frame
Figure 14 The $600^{\text {th }}, 720^{\text {th }}, 840^{\text {th }}, 1000^{\text {th }}, 1200^{\text {th }}$ frame image in original video

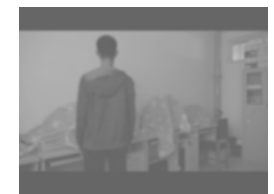

a) $500^{\text {th }}$ frame

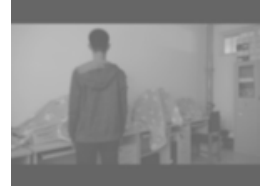

b) $760^{\text {th }}$ frame

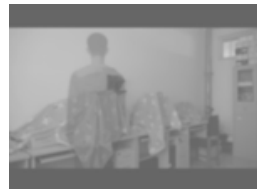

c) $1000^{\text {th }}$ frame

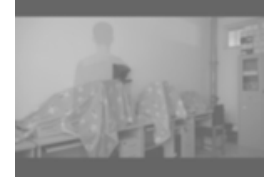

d) $1240^{\text {th }}$ frame

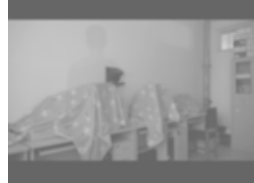

e) $1500^{\text {th }}$ frame
Figure 15 The $600^{\text {th }}, 720^{\text {th }}, 840^{\text {th }}, 1000^{\text {th }}, 1200^{\text {th }}$ frame image in multi frame average method
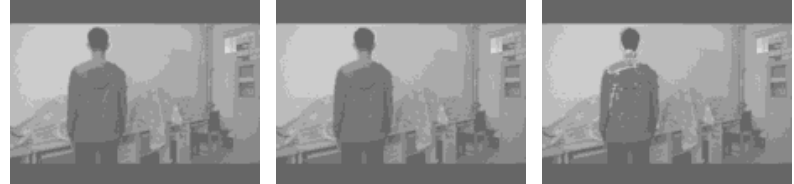

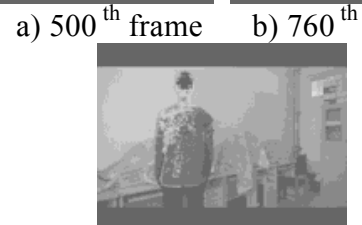

d) $1240^{\text {th }}$ frame

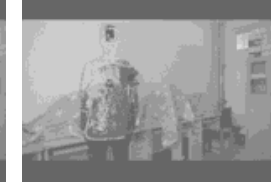

e) $1500^{\text {th }}$ frame
Figure 16 The $600^{\text {th }}, 720^{\text {th }}, 840^{\text {th }}, 1000^{\text {th }}, 1200^{\text {th }}$ frame image in Gauss model

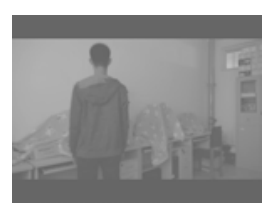

a) $500^{\text {th }}$ frame

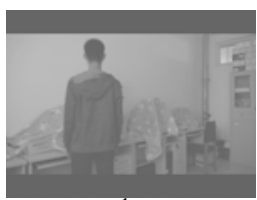

b) $760^{\text {th }}$ frame

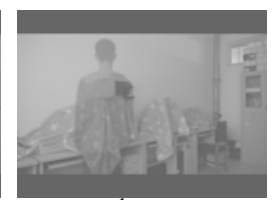

c) $1000^{\text {th }}$ frame

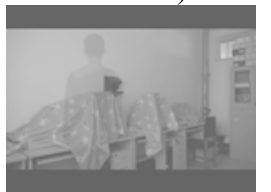

d) $1240^{\text {th }}$ frame

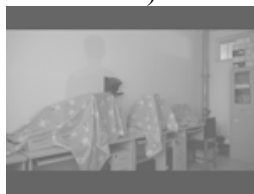

e) $1500^{\text {th }}$ frame
Figure 17 The $600^{\text {th }}, 720^{\text {th }}, 840^{\text {th }}, 1000^{\text {th }}, 1200^{\text {th }}$ frame image in Surendra algorithm

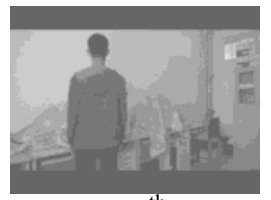

a) $500^{\text {th }}$ frame

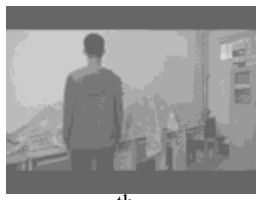

b) $760^{\text {th }}$ frame

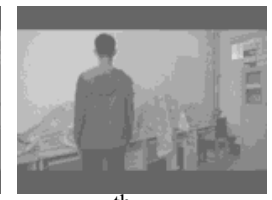

c) $1000^{\text {th }}$ frame

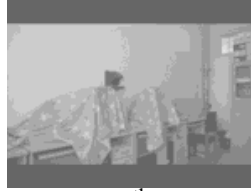

d) $1240^{\text {th }}$ frame

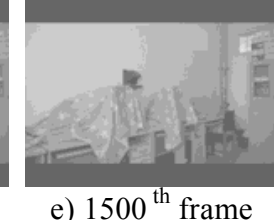

e) $1500^{\text {th }}$ frame
Figure 18 The $600^{\text {th }}, 720^{\text {th }}, 840^{\text {th }}, 1000^{\text {th }}, 1200^{\text {th }}$ frame image in our methods

\section{Conclusion}

The extraction method of static background based on adaptive weight is proposed in this paper. The new parameters of background weights and its variation were introduced. Read by frame respectively, image elements in the current frame are classified after two judgments. The background weights are adjusted firstly. Then the background elements are output through positive and negative judgment of background weight. In our method, the output back- 
ground gray value is relatively accurate, updated quickly, of strong anti-interference ability of the foreground and strong robustness. It is a practical and simple method in principle.

\section{Acknowledgments}

This work is supported by the Specialized Research Fund for the Doctoral Program of Higher Education of China (No. 20122102120004) and Foundation of Liaoning Educational Committee (No. L2012034).

\section{References}

[1] L. Jinju, Z. Qing and W. Yaonan, "Detecting and tracking method of moving target in complex environment," Chinese Journal of Scientific Instrument, Vol. 31, No.10, pp.2242-2247, 2010.

[2] Z. Xuchao et al., "Moving object detection under dynamic background based on phase-correlation and differential multiplication," Chinese Journal of Scientific Instrument, Vol.31, No.5, pp.980-983, 2010.

[3] Lucus B, Kandle T, "An iterative image registration technique with an application to stereo vision," Proceedings of DARPA IU Workshop, pp.121-130, 1981.

[4] L. Changkui, J. Chengyu and W. Ningsheng, "A Novel Statistical Model for Motion Detection and Contour Tracing," Geomatics and Information Science of Wuhan University, Vol.30, No.8, pp.723-727, 2005.

[5] L. Hongwen et al., "The Research of Backgroundsubtraction Based Moving Objects Detection Technology," Journal of National University of Defense technology, Vol.25, No.3, pp.66-69, 2003.

[6] W. Xinyu, Z. Guilin, Study on real-time detection method of moving target based on optical flow," $\mathrm{Co}$ mputer Engineering and Applications, Vol.30, No.1, pp.43-46, 2004.

[7] Stringa E and Regazzoni C S, "Real-time video-shot detection for scene surveilance applications," IEEE Transactions on Image Processing, No.1, pp.69-79, 2000.

[8] L. Bo et al., "ST-CSLBP based space-time mixed background modeling algorithm," Chinese Journal of Scientific Instrument, Vol. 32, No.12, pp. 2781-2786, 2011.

[9] L. Xiying et al., "An algorithm of background extraction and update for saturated intersection," Acta Scientiarum Naturalium Universitatis Sunyatseni, Vol.51, No.3, pp.23-28, 2012. 